\title{
Modelo matemático para planejamento da atracação de navios sob o contrato de Charter Party
}

\author{
Hugo Leonardo Louzada Vervloet ${ }^{1}$ e Rodrigo de Alvarenga Rosa ${ }^{2}$
}

\begin{abstract}
Resumo: Navios de carga granel são usualmente contratados sob o contrato Charter Party entre o armador e o embarcador. Neste contrato é estabelecido um tempo limite de permanência do navio no porto. Caso o navio permaneça abaixo deste tempo, o embarcador recebe um prêmio financeiro e, caso contrário, paga uma multa ao armador. Após a revisão bibliográfica, não foram encontrados artigos sobre o Problema de Alocação de Berços (PAB) que tratassem das questões referentes a prêmio e multa sob a ótica do contrato Charter Party com o objetivo de maximizar o resultado financeiro do embarcador, calculado como prêmio menos multa. Assim, um modelo matemático é proposto para o PAB com considerações sobre a Charter Party e, instâncias baseadas no Porto de Tubarão foram desenvolvidas e executadas no CPLEX 12.6. Os resultados mostraram a aplicabilidade do modelo na programação dos navios e também diretrizes para apoiar a negociação deste tipo de contrato.

Palavras-chave: Problema de Alocação de Berço (PAB), Charter Party, Logística Portuária, Portos Graneleiros.
\end{abstract}

Abstract: Bulk cargo ships are usually contracted under the Charter Party contract between the owner and the shipper. This contract establishes a time limit for vessel to stay at port. If the vessel is below this time the shipper receives a financial award and, if not, pay a fine to the owner. After the literature review, no articles were found on the Berth Allocation Problem (BAP) that addressed the issues related to premium and fine from the perspective of Charter Party contract in order to maximize the financial result of the shipper, calculated as a reward less fine. Thus, a mathematical model is proposed for the BAP with consideration of the Charter Party, and instances based in the Port of Tubarão were developed and implemented in CPLEX 12.6. The results showed the applicability of the model in the scheduling of ships and also guidelines to support the negotiation of this type of contract.

Keywords: Berth Allocation Problem (BAP), Charter Party, Port Logistics, Bulk Ports.

\section{INTRODUÇÃO}

O transporte marítimo desempenha um papel fundamental para o comércio internacional, que integrado a outros sistemas de transportes, representa o meio de transporte de grande parte da carga movimentada no mundo. No modo marítimo, a movimentação de granel sólido em 2013 foi em torno de 10 bilhões de toneladas. $\mathrm{O}$ sistema portuário brasileiro responde por aproximadamente $90 \%$ das exportações e importações no país, sendo que as cargas a granel movimentaram 61\% da movimentação total em 2014 (UNCTAD, 2014).

Os navios de granel, como minério de ferro e agrícolas, são usualmente contratados sob o contrato de Charter Party entre o armador (dono do navio) e o embarcador. As cargas a granel são usualmente transportadas em grande quantidade e, assim, muitas empresas que comercializam estas cargas optam por ter seus próprios portos ou por operarem terminais portuários específicos para suas cargas. A partir deste ponto, define-se para efeitos deste artigo que quando se referenciar ao embarcador, entende-se por um embarcador que é proprietário do porto ou um embarcador operador de um terminal específico dedicado às suas operações.

\footnotetext{
${ }^{1}$ Hugo Leonardo Louzada Vervloet, Programa de Pós-Graduação em Engenharia Civil, Universidade Federal do Espírito Santo, Vitória, ES, Brasil (hugo.vervloet@gmail.com).

2 Rodrigo de Alvarenga Rosa, Programa de Pós-Graduação em Engenharia Civil, Universidade Federal do Espírito Santo, Vitória, ES, Brasil (rodrigo.a.rosa@ufes.br).

Manuscrito recebido em 13/08/2015 e aprovado para publicação em 24/02/2016.

Este artigo é parte de TRANSPORTES v. 24, n. 1, 2016 ISSN: 2237-1346 (online). DOI: 10.14295/transportes.v24i1.976
}

Para atender aos interesses dos armadores e dos embarcadores, no contrato de Charter Party são definidos: um período de tempo para chegada do navio e um período de tempo para operar o navio no porto. O período de tempo para chegada do navio no porto é denominado layday e caso o navio chegue ao porto e esteja apto para operar neste período, ele terá prioridade de atendimento. O prazo que o porto tem para operar o navio é denominado laytime. O laytime passa a contar a partir de uma data marco denominada Aceite, que será mais detalhada na Seção 2, sendo que este Aceite é dado pelo porto em função do navio chegar dentro do período de Layday ou fora deste período. O tempo de permanência do navio no porto é calculado como sendo a soma do tempo de espera para atracar a partir da data do Aceite mais o tempo de operação. Caso o tempo de permanência seja menor do que o prazo máximo estabelecido no contrato, Laytime, o embarcador tem direito a receber um valor financeiro denominado Prêmio, dispatch, e, caso contrário, cabe ao embarcador pagar um valor financeiro ao armador denominado Multa, demurrage. Tendo em vista que o embarcador é o proprietário do porto ou o operador do porto, ele visa obter resultados positivos, ou seja, pagar o menor valor de multa e receber o maior volume de prêmio a fim de ter um resultado positivo. Assim sendo, o embarcador estende as relações contratuais operacionais constantes da Charter Party para as metas operacionais do porto (Wilson, 1988).

Muitos embarcadores convivem com significativos impactos financeiros negativos gerados pelo pagamento de Multa por não conseguirem operar os navios dentro dos tempos estabelecidos nos contratos de Charter Party. Assim, neste artigo, que se considera o embarcador proprietário ou 
operador do porto, um bom planejamento da sequência de atracação dos navios poderia, eventualmente, ser revertido em recebimento de Prêmio. No entanto, a regra mais utilizada na relação porto e navios é a First Come First Served (FCFS) onde a ordem de atracação é definida pela ordem de chegada do navio ao porto. O que se propõe neste artigo é a análise da flexibilização desta regra e, assim, elaborar a sequência de atendimento aos navios visando a melhoria do resultado financeiro do embarcador advinda da relação do total de prêmio recebido menos o total de multa paga.

Para planejar a atracação de navios no porto, é proposto na literatura cientifica o Problema de Alocação de Berços (PAB) que trata do problema de planejar a sequência de atendimento dos navios em cada berço do porto, em um certo horizonte de tempo de planejamento, atendendo às restrições operacionais do porto. O principal objetivo do PAB nos artigos publicados é minimizar o tempo de permanência dos navios no porto (Bierwirth e Meisel, 2010). Diversos artigos foram publicados sobre o tema, dentre eles, destacam-se as revisões realizadas por Meersmans e Dekker (2001), Vis e Koster (2003), Steenken et al. (2004), Vacca et al. (2007), Stahlbock e Voss (2008), Bierwirth e Meisel (2010) e Rashidi e Tsang (2013). Apesar da importância do contrato de Charter Party, tendo em vista a grande quantidade de navios a granel que são contratados por meio deste tipo de contrato e pelo volume de multa pago pelos embarcadores aos armadores, após a revisão da literatura, Seção 2, pôde-se perceber que nenhum artigo abordou as questões relativas ao contrato de Charter Party como função objetivo e/ou restrição de um modelo PAB.

Este artigo propõe um modelo matemático para o PAB com foco nos navios sob o contrato de Charter Party, principalmente em portos graneleiros, com o objetivo de elaborar a sequência de atracação dos navios visando à melhoria do resultado financeiro do embarcador, que neste artigo é também o proprietário do porto, calculado como sendo a diferença entre os valores recebidos de prêmio do armador menos os valores pagos de multa ao armador. O modelo proposto considera todos os itens contratuais, tempo de Layday, chegada ao porto, Aceite, atracação, Laytime e desatracação para poder calcular os valores de prêmio e multa.

Para avaliar o modelo proposto, o mesmo foi testado com dados do Porto de Tubarão, localizado em Vitória/ ES, um dos maiores portos mundiais especializados na movimentação de granéis minerais, sendo que a totalidade dos navios é operada em contrato de Charter Party e a empresa proprietária do porto é o embarcador da maioria das cargas. O porto opera mais de 600 navios por ano com uma movimentação superior a 100 milhões de toneladas por ano. Possui três berços de carregamento de minério de ferro e pelotas, sendo eles: Píer 1 Sul, Píer 1 Norte e Píer 2 (Vale, 2015).

O presente artigo está organizado com a seguinte estrutura. Na Seção 2 é abordado o contrato de Charter Party, a explicação do cálculo de prêmio e multa, a definição do Problema de Alocação de Berço (PAB) e uma revisão da literatura sobre o PAB. A Seção 3 apresenta o modelo matemático proposto. Na Seção 4 são apresentados os resultados e as análises e, por fim, a Seção 5 apresenta a conclusão deste artigo.

\section{REFERENCIAL TEÓRICO}

O transporte marítimo é influenciado diretamente pela oferta e demanda do mercado e, para estabelecer limites entre os direitos e responsabilidades das partes, são realizados contratos onde o armador se compromete a disponibilizar sua embarcação para transporte de mercadorias pertencentes a uma ou mais pessoas, chamado contrato de afretamento. Um dos instrumentos mais importantes utilizado para formalizar o afretamento de um navio é denominado Charter Party (Wilson, 1988).

O Charter Party é um contrato bilateral de afretamento de navio, por viagem ou período de tempo, e mundialmente o mais utilizado no transporte marítimo de carga a granel. Nos contratos Charter Party são estabelecidos acordos e condições do que variam conforme a modalidade de contratação. Podem-se citar: o valor do frete, as condições de pagamento, o período do afretamento, os tempos para carregamento e descarga, o tipo e a quantidade de mercadoria a serem transportados, os portos de origem e destino, as condições de operação e o prêmio e multa (Stopford, 1997).

A Charter Party pode ser firmada entre o armador e o embarcador de três formas: 1) Voyage Charter; 2) Time Charter e 3) Bare Boat. Na Voyage Charter, o navio é contratado pelo embarcador para realizar uma viagem entre um porto de origem e um porto de destino para transportar uma certa carga. Na Time Charter, o navio é contratado pelo embarcador por um período de tempo, usualmente anos, e, então o embarcador contratante pode realizar diversas viagens neste período. Na Bare Boat ocorre uma situação diferente onde o navio é arrendado a uma outra empresa a casco nu, este tipo de Charter Party não será tratado neste artigo. $\mathrm{O}$ maior foco deste artigo são as Charter Party na forma Voyage Charter e, também, o modelo proposto no artigo trata dos quesitos da Time Charter.

Na Voyage Charter existem algumas definições importantes, tais como: Layday e Laytime, que são explicadas a seguir. O período de layday estabelece um período dentro o qual o navio deverá chegar ao porto e estar disponível para operar. Quanto maior for o período de layday, maior a incerteza de quando o navio poderá chegar ao porto. $\mathrm{O}$ laytime é o prazo que o armador do navio se dispõe a mantê-lo à disposição do porto para as operações de carga e/ou descarga, sem a necessidade de pagamento extra para este período. O tempo de operação de carregamento ou descarga do navio varia em função de algumas atividades: manobra para atracação e desatracação, do preparo da documentação e preparo da carga que será movimentada. Varia também em função das características do berço, devido à capacidade dos equipamentos existentes (Wilson, 1988).

Nos contratos de Charter Party do tipo Voyage Charter, a principal penalidade utilizada na relação entre o embarcador e o armador é chamada demurrage ou multa. Trata-se de uma compensação financeira paga pelo embarcador ao armador caso o tempo que o navio ficou à disposição do porto ultrapasse o laytime acordado em contrato (Collyer, 2006). O embarcador deve negociar um período de laytime suficiente para as operações de carga e/ou descarga.

Ainda no contrato Charter Party do tipo Voyage Charter, em contrapartida à penalidade e com o intuito de premiar o embarcador por ter operado o porto em um tempo 


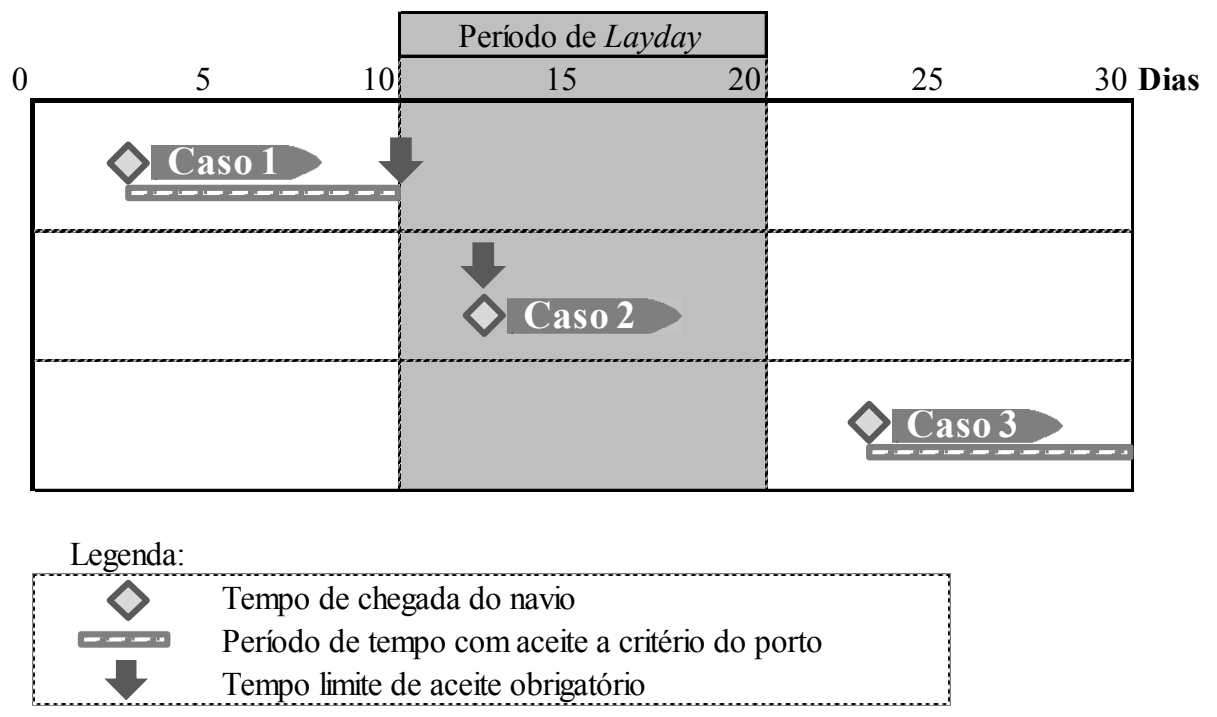

Figura 1: Utilização prática do período de layday

inferior ao laytime, o armador remunera o embarcador com o prêmio ou dispatch. Internacionalmente, como prática de mercado, o porto e o armador acordam em contrato o valor de prêmio como a metade do valor acordado de multa. Porém existem negociações onde o valor de prêmio e multa são iguais e também há casos em que não há uma regra de proporcionalidade.

Para cálculo da multa e do prêmio, a primeira informação a ser conhecida é o momento que o navio chegou no porto. Três situações podem ocorrer, o navio chegou antes, durante ou depois do período de layday. Na Figura 1 podem ser vistas as três situações.

Na Figura 1, no Caso 1, quando o navio chega ao porto antes do início do período de layday, o embarcador pode optar em dar o aceite ao navio antes do período de layday, pois a partir do Aceite começa a incorrer o laytime. Caso o embarcador não dê o Aceite antes do período de layday, ele deve obrigatoriamente dar o Aceite ao navio no tempo de início do período de layday. Na Figura 1, Caso 2, quando o navio chega dentro do período de layday, o embarcador necessariamente deve emitir o aceite ao navio no momento da chegada do navio no porto, mesmo que o navio não seja operado de imediato.

$\mathrm{Na}$ Figura 1, Caso 3, quando o navio chega após o período de layday, o embarcador pode optar em dar o Aceite a qualquer momento posterior à chegada do navio. Usualmente, o embarcador emite o Aceite no momento que o porto está disponível para atracar o navio, evitando assim qualquer pagamento de multa. Neste caso, o Aceite do embarcador e o início da contagem do laytime se darão no momento da atracação do navio.

O tempo de permanência do navio no porto é calculado como sendo o tempo de término da operação do navio no porto subtraído do tempo de Aceite. Se este tempo de permanência for maior que o laytime acordado, o embarcador pagará multa ao navio, caso seja menor, o embarcador receberá prêmio do armador e caso seja zero não haverá multa nem prêmio na operação do navio.

Como no porto em estudo neste artigo o embarcador é o proprietário do porto, ele estende estes quesitos opera- cionais da Charter Party como metas operacionais do porto visando obter um melhor resultado financeiro obtendo mais prêmio que multa na sua relação com os armadores.

Nos contratos de Charter Party do tipo Time Charter não há a cobrança de Prêmio e Multa. No entanto, vários trades, dentre eles citam-se: minérios, agrícolas, dentre outros, podem eventualmente elaborar contratos paralelos à Time Charter para definir um período de laydays e o laytime para os navios entre o embarcador, o armador e o porto a fim de se estabelecer níveis de serviço do porto. Com base neste contrato paralelo, o embarcador define o Aceite de um navio sob o contrato Charter Party do tipo Time Charter da mesma forma explicada anteriormente para um contrato Charter Party do tipo Voyage Charter. Vale ressaltar que isto é uma excepcionalidade comercial e não faz parte da Charter Party.

$\mathrm{Na}$ literatura cientifica é proposto o Problema de Alocação de Berços (PAB) que tem por objetivo elaborar a sequência de atendimento aos navios que virão a operar no porto no conjunto de berços disponíveis em um horizonte de tempo de planejamento respeitando as restrições temporais e espaciais do porto (Cordeau et al., 2005). A função objetivo do PAB na maior parte dos problemas visa reduzir a permanência do navio no porto (Bierwirth e Meisel, 2010).

A restrição temporal trata da chegada dos navios de duas formas. Na chegada estática, os navios já estão disponíveis para atracação, ou seja, os navios já estão no porto. Na chegada dinâmica, os navios possuem datas de chegada ao longo do horizonte de planejamento e não podem atracar antes da sua data de chegada ao porto (Imai et al., 2001).

A restrição espacial diz respeito ao calado, comprimento e boca do navio em relação às características equivalente do porto. Quanto ao layout do cais, o PAB pode ser dividido em PAB discreto (PABD), PAB contínuo (PABC) e PAB híbrido (PABH). No PABD um cais é segmentado em berços, onde cada navio pode ocupar apenas um determinado berço e o navio deve ter comprimento menor que o comprimento do berço. No PABC o cais não possui segmentação em berços e, portanto, os navios podem ser atra- 
cados em qualquer posição livre do cais. Rodrigues et al. (2015) analisaram o PABC onde não divisão do cais, porém há restrições de atracações pela descontinuidade do cais. $\mathrm{O}$ $\mathrm{PABH}$ possui as características tanto do PABD quanto do PABC, permitindo que navios ocupem mais de um berço ou que mais de um navio ocupe um berço (Bierwirth e Meisel, 2010; Imai et al., 2005).

Imai et al. (2001) propuseram a introdução de janelas de atracação para portos cujos navios possuem chegada dinâmica e os berços, taxas de produtividade distintas. Hansen e Oguz (2003), Theofanis et al. (2007) e Imai et al. (2008) solucionam o PAB a partir da atribuição e sequenciamento dos navios em berços, como objetivo de minimizar o tempo de espera e a operação dos navios. Imai et al. (2003) realizaram estudos de PABD com chegada dinâmica, assim como Monaco e Sammarra (2007). Nishimura et al. (2001) analisaram o problema de um porto público com berços discretos, que recebe navios com chegada dinâmica e que podem atracar em qualquer berço do porto. Eles propuseram um modelo matemático não linear para o problema e, como o modelo é de difícil solução, eles propuseram também a utilização de Algoritmo Genético. Zhou et al. (2008) e Han et al. (2010) consideraram chegada e o tempo de operação do navio como um comportamento estocástico. Cordeau et al. (2005) propuseram um modelo baseado no Problema de Roteamento de Veículos multidepósitos com janela de tempo (PRV-MD), onde os navios são vistos como clientes e os berços como depósitos/veículos e utilizaram a meta-heurística Tabu Search. Golias et al. (2006) e Golias (2007) consideraram como variáveis estocásticas os horários de chegada e tempos de movimentação de navios. Golias et al. (2010) propuseram um Algoritmo Genético para o PABD com o objetivo minimizar o consumo de combustível dos navios.

Hansen e Oguz (2003) e Hansen et al. (2008) propuseram o uso da meta-heurística Variable Neighborhood Search (VNS) que apresentou resultados superiores aos encontrados por Nishimura et al. (2001). Han et al. (2006) e Zhou et al. (2006) utilizaram Algoritmo Genético para resolver o problema que considera a chegada dos navios como estocástica e restrição do tempo de espera. Imai et al. (2008) propuseram a utilização do Algoritmo Genético com o objetivo de minimizar os navios rejeitados, ou seja, os navios que não são atendidos dentro do prazo máximo acordado.

Considerando como base o trabalho elaborado por Cordeau et al. (2005), Mauri et al. (2008) utilizaram uma abordagem baseada em Geração de Colunas e apresentaram uma melhora no tempo de execução para encontrar as soluções. Mauri et al. (2010) trataram o PABD como um PRV-MD utilizando a meta-heurística Algorithm Training Population / Linear Programming (PTA/LP).

Lorenzoni et al. (2006) desenvolveram um modelo matemático com base no problema de escalonamento para solucionar o problema de alocação de recursos para atender os navios de minério de ferro, granel, visando reduzir o pagamento de multas. No entanto, eles não se preocuparam em elaborar a sequência de atendimento dos navios e optaram por manter a regra First Come First Served (FCFS), ou seja, o primeiro navio a chegar é o primeiro navio a ser atendido. Em contrapartida, o modelo proposto neste artigo tem por hipótese a flexibilização da regra FCFS e, a partir desta flexibilização buscar definir uma sequência ideal para atracação dos navios considerando os tempos de chegada do navio e as cláusulas da Charter Party com enfoque operacional. O objetivo do modelo proposto neste artigo é similar ao de Lorezoni et al. (2006), ou seja, a maximização do resultado financeiro. Mas neste ponto, os artigos também diferem, pois no modelo proposto por eles são considerados somente os valores pagos de multa e não é levado em conta valores recebidos de prêmio, enquanto no modelo proposto neste presente artigo leva em conta o resultado financeiro advindo dos valores recebidos de prêmio menos os valores pagos de multa.

Buhrkal et al. (2011) realizaram um comparativo entre o PABD, PABC e PABH, utilizando uma perspectiva computacional por meio de extensos testes numéricos. Ting et al. (2013) formularam um modelo de Programação Inteira Mista (PIM) a partir da atribuição dos navios aos berços e com o objetivo de minimizar o tempo total de espera e o tempo de manuseio para os navios. Rosa et al. (2013) sugeriram a criação do PAB Múltiplas Cargas (PAB-MC), por meio de um modelo matemático baseado em PLIM. Teixeira e Cunha (2012) analisaram a distribuição de contêineres vazios e seu impacto no porto. Banos et al. (2016) propuseram para o mesmo problema tratado por Rosa et al. (2012) um novo modelo matemático e uma meta-heurística Simulated Annealing.

\section{MODELO MATEMÁTICO PROPOSTO}

O modelo matemático proposto visa planejar a sequência de atendimento dos navios considerando questões de um contrato Charter Party tais como: Layday, Laytime, Aceite, Atracação e Desatracação. O modelo tem por objetivo maximizar o resultado financeiro gerado pelo porto por meio da subtração entre o valor de prêmio recebido e o valor da multa paga pelo porto aos armadores dos navios.

O modelo proposto pode ser classificado como um PAB com berço discreto, chegada dinâmica (restrição temporal pela instante de chegada do navio no porto) e com restrições espaciais, o deadweight do navio em relação ao dea$d$ weight admitido em cada berço. $\mathrm{O}$ modelo proposto tem por objetivo tratar principalmente dos navios com contrato Voyage Charter. O modelo proposto neste artigo e apresentado nesta seção teve como inspiração o problema Job Shop Scheduling. O modelo matemático proposto é apresentado em cinco partes: conjuntos, parâmetros, variáveis de decisão, função objetivo e restrições.

\section{Conjuntos}

$N$ Conjunto de navios para chegar ao porto, $i=1 \ldots n$;

$N 0$ Conjunto de navios $N$ adicionado de um navio fictício, que permitirá modelar a sequência de atracação, $i=1 . . . n$, considerando o 0 como origem do fluxo;

$B$ Conjunto de berços disponíveis no porto, $k=1 . . . b$;

\section{Parâmetros}

Em relação ao navio $i \in N$, têm-se:

$s_{i}$ Tempo do início de layday;

$e_{i}$ Tempo do término de layday;

$l_{i}$ Prazo (tempo) de laytime;

$\eta_{i}$ Tonelagem de porte bruto;

$m_{i}$ Valor da multa por hora; 
$p_{i}$ Valor do prêmio por hora;

$a_{i}$ Instante de chegada;

$o_{i k}$ Tempo de operação no berço $k \in B$.

Para o berço $k \in B$ tem-se:

$b_{k}$ Tonelagem de porte bruto máximo admitido;

E por fim, tem-se:

$M$ Parâmetro para a lógica do modelo que tem por função ativar ou desativar certas restrições ao longo do modelo, foi adotado um valor muito grande, no caso 999.999.

\section{Variáveis de decisão}

Em relação ao navio $i \in N$, têm-se as seguintes variáveis de decisão:

$\delta_{i} \quad$ Instante do aceite;

$\tau_{i k}$ Tempo de término da operação no berço $k \in B$;

$\alpha_{i k}$ Período em que incide o pagamento de prêmio (dispatch) no berço $k \in B$;

$\beta_{i k}$ Período em que incide o pagamento de multa (demurrage) no berço $k \in B$;

Têm-se ainda as duas variáveis de controle que são:

$$
x_{i j k}\left\{\begin{array}{l}
1 \text { Se o navio } i \in N 0 \text { precede imediatamente ao } \\
\text { navio } j \in N 0 \text { no berço } k \in B \\
0 \text { Caso contrário. }
\end{array}\right.
$$

\section{Se o tempo de permanência do navio $i \in N$ no berço $k \in B$ é superior ao laytime, ou seja, se a \\ $Z_{i k}\{$ operação gerou multa para o porto; 1 Se o tempo de permanência do navio $i \in N$ no berço $k \in B$ é igual ou inferior ao laytime, ou seja, se a operação gerou prêmio para o porto;}

A função objetivo, Equação (1), representa a maximização do valor recebido de prêmio menos o valor pago de multa por todos os navios operados pelo porto. A função objetivo é estabelecida por duas parcelas. A primeira parcela representa o valor de prêmio calculado como a multiplicação da taxa de prêmio de cada navio pelo valor de prêmio do navio. A segunda parcela representa o valor de multa calculado como a multiplicação da taxa de multa de cada navio pelo valor de multa.

As Restrições (2) asseguram uma sequência de atracação onde cada navio $j \in N$ tenha um único navio $i \in N$ predecessor imediato em um único berço $k \in B$. As Restrições (3) garantem que cada berço $k \in B$, se usado, tenha uma única sequência de processamento, onde o 0 é o início de atendimento. As Restrições (4) garantem que cada navio $h \in N$ possua somente um navio posterior a ele no berço $k \in B$. As Restrições (5) garantem que o instante de término no berço $k \in B$ do navio $j \in N$ seja igual ao instante de término do navio $i \in N$ mais o tempo de operação do navio $j \in N$.

As Restrições (6) garantem que todos os berços $k \in B$ começam a operar no tempo 0 . As Restrições (7) garantem que os navios $i \in N$ sejam atendidos somente após sua chegada ao porto, enquanto que as Restrições (8) garantem que os navios $i \in N$ somente receberão o aceite $\delta_{i}$ após o instante de chegada $a_{i}$ do navio $i \in N$ no porto.
As Restrições (9) garantem que se o navio $i \in N$ chegar ao porto dentro do período de layday $\left(s_{i} \leq a_{i} \leq e_{i}\right)$, o aceite $\delta_{i}$ do navio $i \in N$ pelo porto ocorrerá no tempo de chegada $a_{i}$. As Restrições (10) asseguram que se o navio $i \in N$ chegar ao porto antes do início de layday $a_{i} \leq s_{i}$, o aceite $\delta_{i}$ ficará a critério do porto e poderá ocorrer entre a chegada do navio $i \in N$ e o tempo de inicio de layday $s_{i}$. As Restrições (11) asseguram que se o navio $i \in N$ chegar ao porto após o fim de layday $\left(a_{i} \geq e_{i}\right)$ o aceite $\delta_{i}$ do navio $i \in N$ ficará a critério do porto e será considerado como o tempo do início da operação do navio $\left(\tau_{i k}-O_{i k}\right)$.

As Restrições (12) e as Restrições (13) em conjunto definem se a operação gerou prêmio $Z_{i k}=1$ ou multa $\mathrm{Z}_{i k}=0$ para o porto. Para tanto, é calculado o prazo de laytime menos o tempo de permanência que é calculado como sendo o tempo de término da operação menos o tempo de aceite.

As Restrições (14) definem que para os navios $i \in N$ que forem receber prêmio, $Z_{i k}=1$, o período de prêmio $\alpha_{i k}$ do navio $i \in N$ no berço $k \in B$ seja menor ou igual que o laytime $l_{i}$ menos o tempo de término da operação $\tau_{i k}$ do navio $i \in N$ mais o aceite do navio $i \in N$. As Restrições (15) garantem que para os navios $i \in N$ que não forem atracar no berço $k \in B$, o período de prêmio $\alpha_{i k}$ do navio $i \in N$ atracado no berço seja menor ou igual à zero, caso o navio $i \in N$ atraque no berço $k \in B$, então, o período de prêmio do navio $i \in N$ no berço $k \in B$ pode ser menor ou igual a um valor muito grande $M$. As Restrições (16) garantem que para os navios $i \in N$ que não recebem prêmio no berço $k \in B, \mathrm{Z}_{i k}=0$, o período de prêmio $\alpha_{i k}$ do navio $i \in N$ atracado no berço $k \in B$ seja menor ou igual à zero, caso o navio receba prêmio, $Z_{i k}=0$, no berço $k \in B$, então, o período de prêmio pode ser menor ou igual a um valor muito grande $M$.

As Restrições (17) definem que quando o navio $i \in N$ receber multa, $\mathrm{Z}_{i k}=0$, o período de multa $\beta_{i k}$ do navio $i \in N$ no berço $k \in B$ seja maior ou igual que o tempo de término da operação $\tau_{i k}$ do navio $i \in N$ menos o aceite $\delta_{i}$ do navio $i \in N$ menos o laytime $l_{i}$. As Restrições (18) garantem que se o navio $i \in N$ não atracar no berço $k \in B$, o período de multa $\beta_{i k}$ do navio $i \in N$ atracado no berço $k \in B$ seja menor ou igual a zerocaso o navio $i \in N$ atraque no berço $k \in B$, então, o período de multa do navio $i \in N$ no berço $k \in B$ será menor ou igual que um valor muito grande $M$. As Restrições (19) garantem que os navios que não recebem multa, $Z_{i k}=1$, tenham o período de multa $\beta_{i k}$ do navio $i \in N$ atracado no berço $k \in B$ menor ou igual a zero e, caso o navio receba multa, $\mathrm{Z}_{i k}=0$, então, o período de multa será menor ou igual a um valor muito grande $M$.

As Restrições (20) garantem que o berço tenha capacidade para atracação $b_{k}$ do navio $i \in N$. As Restrições (21) garantem que o tempo de término da operação $\tau_{i k}$ do navio seja maior ou igual à zero. As restrições (22) garantem que o período de prêmio $\alpha_{i k}$ do navio $i \in N$ no berço $k \in B$ seja maior ou igual à zero. As Restrições (23) garantem que o período de multa $\beta_{i k}$ do navio $i \in N$ no berço $k \in B$ seja maior ou igual à zero. As Restrições (24) estabelecem que a variável $x_{i j k}$ é binária. As Restrições (25) garantem que a variável $Z_{i k}$ seja binária (0 ou 1). 


\section{Função Objetivo}

Minimizar $\sum_{i \in N} \sum_{k \in B}\left(\alpha_{i k} p_{i}\right)-\sum_{i \in N} \sum_{k \in B}\left(\beta_{i k} m_{i}\right)$

Restrições

$$
\begin{aligned}
& \sum_{k \in B} \sum_{i \in N 0} x_{i j k}=1 \\
& \forall j \in N \\
& \sum_{j \in N} x_{0 j k} \leq 1 \\
& \forall k \in B \\
& \sum_{\substack{i \in N 0 \mid \\
i \neq h}} x_{i h k}-\sum_{\substack{j \in N 0 \mid \\
j \neq h}} x_{h j k}=0 \\
& \forall h \in N, k \in B \\
& \tau_{j k} \geq \tau_{i k}-M+\left(o_{j k}+M\right) x_{i j k} \\
& \forall i \in N 0, j \in N, k \in B \\
& \tau_{0 k}=0 \\
& \forall k \in B \\
& \tau_{i k} \geq\left(a_{i}+o_{i k}\right) \sum_{j \in N 0} x_{i j k} \\
& \forall i \in N, k \in B \\
& \delta_{i} \geq a_{i} \\
& \forall i \in N \\
& \delta_{i}=a_{i} \\
& \forall i \in N, s_{i} \leq a_{i} \leq e_{i} \\
& \delta_{i} \leq s_{i} \\
& \delta_{i} \geq \tau_{i k}-o_{i k}-\left(1-\sum_{j \in N 0} x_{i j k}\right) M \\
& \forall i \in N, k \in B, a_{i} \leq s_{i} \\
& \forall i \in N, k \in B, a_{i} \geq e_{i} \\
& l_{i}-\left(\tau_{i k}-\delta_{i}\right)-z_{i k} M \leq 0 \\
& \forall i \in N, k \in B \\
& l_{i}-\left(\tau_{i k}-\delta_{i}\right)+\left(1-z_{i k}\right) M \geq 0 \\
& \forall i \in N, k \in B \\
& \alpha_{i k} \leq\left(l_{i}-\left(\tau_{i k}-\delta_{i}\right)\right)+\left(1-z_{i k}\right) M \\
& \forall i \in N, k \in B \\
& \alpha_{i k} \leq M \sum_{j \in N 0} x_{i j k} \\
& \forall i \in N, k \in B \\
& \alpha_{i k} \leq M z_{i k} \\
& \forall i \in N, k \in B \\
& \beta_{i k} \geq\left(\left(\tau_{i k}-\delta_{i}\right)-l_{i}\right)-z_{i k} M \geq 0 \\
& \forall i \in N, k \in B \\
& \beta_{i k} \leq M \sum_{j \in N 0} x_{i j k} \\
& \forall i \in N, k \in B \\
& \beta_{i k} \leq\left(1-z_{i k}\right) M \\
& \forall i \in N, k \in B \\
& \beta_{i k} \geq \eta_{i} \sum_{j \in N 0} x_{i j k} \\
& \forall i \in N, k \in B \\
& \tau_{i k} \geq 0 \\
& \forall i \in N 0, k \in B \\
& \alpha_{i k} \geq 0 \\
& \forall i \in N, k \in B \\
& \beta_{i k} \geq 0 \\
& \forall i \in N, k \in B \\
& x_{i j k} \in\{0,1\} \\
& \forall i \in N 0, j \in N 0, k \in B \\
& z_{i k} \in\{0,1\} \\
& \forall i \in N, k \in B
\end{aligned}
$$


Como definido anteriormente, sendo $n$ o número de navios e $b$ o número de berços, tem-se que o modelo matemático proposto possui $n+n^{2} b+4 n b$ variáveis e no máximo $2 n+4 b+21 n b+2 n^{2} b$ restrições.

\section{TESTES COMPUTACIONAIS E ANÁLISE DOS RESULTADOS}

Para avaliar o modelo matemático proposto, os dados operacionais do Porto de Tubarão foram obtidos na Praticagem do ES (Praticagem, 2015) e correspondem ao mês de junho de 2015. As informações operacionais obtidas foram: navio, data de chegada, volume movimentado, tempo de operação e berço utilizado. As informações comerciais, período de layday, laytime, e os valores de prêmio e multa, foram formuladas com referencias de mercado obtidas de dados históricos do Porto de Tubarão. A fim de conservar a confidencialidade das informações, todos os dados foram multiplicados aleatoriamente por um fator de ajuste. Considerou-se um horizonte de planejamento de 15 dias.

As instâncias foram agrupadas em quatro grupos para avaliar o impacto das variações Layday, Laytime, Tempo de operação e percentual de valor entre prêmio e multa. Todos esses valores são estabelecidos diretamente ou indiretamente no contrato de Charter Party. O Grupo A tem quatro instâncias que testam o impacto financeiro em função da variação do período de Layday, foram testados períodos com 5, 10, 15 e 20 dias. Dos resultados deste grupo, adotou-se a Instância 2 como referência para os testes dos outros grupos, pois ela apresentou os melhores resultados e tem as características mais próximas da realidade do Porto de Tubarão.

O Grupo B tem quatro instâncias que testam o impacto financeiro em função da variação do período de Laytime, foram utilizados tempos com $+10 \%,+20 \%,+30 \%$ e $+40 \%$ de horas em relação à Instância 2. $\mathrm{O}$ Grupo $\mathrm{C}$ tem quatro instâncias que testam o impacto financeiro em função da variação do tempo de operação, foram testados tempos com $-10 \%,-20 \%,-30 \%$ e $-40 \%$ de horas em relação à Instância 2. Por fim, o Grupo D, com três instâncias, avalia o impacto da variação entre o valor de prêmio e multa, variou-se a relação entre o valor de prêmio e multa nas seguintes razões: $25 \%, 75 \%$ e $100 \%$ da Multa em relação à Instância 2.

Assim, as instâncias de teste podem ser vistas na Tabela 1 onde a coluna Grupo informa o grupo das instâncias que serão analisadas. A coluna Instância informa o número da instância. A coluna Número de Berços informa a quantidade de berços considerada. A coluna Número de Navios informa a quantidade de navios considerada. A coluna Layday informa o período de Layday adotado. $\mathrm{Na}$ coluna Laytime são apresentados os valores considerados de laytime. A coluna Tempo de Carregamento apresenta o tempo de carregamento adotado por navio para cada berço. Na coluna Valor do Prêmio é informado o percentual do valor do prêmio em relação ao valor da multa. A especificação Padrão na Tabela 1 em qualquer coluna re-

Tabela 1: Instâncias desenvolvidas para teste do modelo proposto

\begin{tabular}{|c|c|c|c|c|c|c|c|}
\hline Grupo & Instância & $\begin{array}{l}\text { Número de } \\
\text { Berços }\end{array}$ & $\begin{array}{l}\text { Número de } \\
\text { Navios }\end{array}$ & $\begin{array}{l}\text { Layday } \\
\text { (dia) }\end{array}$ & Laytime & $\begin{array}{l}\text { Tempo de } \\
\text { Carregamento }\end{array}$ & $\begin{array}{l}\text { Valor do } \\
\text { Prêmio }\end{array}$ \\
\hline \multirow{5}{*}{$\mathbf{A}$} & 1 & \multirow{5}{*}{3} & \multirow{5}{*}{25} & 5 & \multirow{5}{*}{ Padrão } & \multirow{5}{*}{ Padrão } & \multirow{5}{*}{ Padrão } \\
\hline & 2 & & & 10 & & & \\
\hline & & & & & & & \\
\hline & 3 & & & 15 & & & \\
\hline & 4 & & & 20 & & & \\
\hline \multirow{5}{*}{ B } & 5 & \multirow{5}{*}{3} & \multirow{5}{*}{25} & \multirow{5}{*}{ Padrão } & Padrão + $10 \%$ & \multirow{5}{*}{ Padrão } & \multirow{5}{*}{ Padrão } \\
\hline & 6 & & & & Padrão + $20 \%$ & & \\
\hline & & & & & & & \\
\hline & 7 & & & & Padrão +30\% & & \\
\hline & 8 & & & & Padrão +40\% & & \\
\hline \multirow{5}{*}{ C } & 9 & \multirow{5}{*}{3} & \multirow{5}{*}{25} & \multirow{5}{*}{ Padrão } & \multirow{5}{*}{ Padrão } & Padrão - $10 \%$ & \multirow{5}{*}{ Padrão } \\
\hline & 10 & & & & & Padrão -20\% & \\
\hline & & & & & & & \\
\hline & 11 & & & & & Padrão -30\% & \\
\hline & 12 & & & & & Padrão - $40 \%$ & \\
\hline \multirow{3}{*}{ D } & 13 & \multirow{3}{*}{3} & \multirow{3}{*}{25} & \multirow{3}{*}{ Padrão } & \multirow{3}{*}{ Padrão } & \multirow{3}{*}{ Padrão } & $25 \%$ da Multa \\
\hline & 14 & & & & & & $75 \%$ da Multa \\
\hline & 15 & & & & & & $100 \%$ da Multa \\
\hline
\end{tabular}


Tabela 2: Resultados apresentados pelo CPLEX para as instâncias desenvolvidas

\begin{tabular}{|c|c|c|c|c|c|c|c|c|}
\hline \multicolumn{9}{|c|}{ CPLEX } \\
\hline Grupo & Instância & $\begin{array}{r}\text { Função } \\
\text { Objetivo } \\
(R \$)\end{array}$ & $\begin{array}{r}\text { Lower } \\
\text { Bound } \\
\text { (R\$) }\end{array}$ & $\begin{array}{r}\text { Upper } \\
\text { Bound } \\
\text { (R\$) }\end{array}$ & $\begin{array}{r}\text { GAP } \\
(\%)\end{array}$ & $\begin{array}{r}\text { Tempo de } \\
\text { Processa-mento } \\
\text { (s) }\end{array}$ & $\begin{array}{r}\text { Prêmio } \\
\text { (R\$) }\end{array}$ & $\begin{array}{r}\text { Multa } \\
\text { (R\$) }\end{array}$ \\
\hline \multirow{4}{*}{ A } & 1 & 67.707 & 67.707 & 67.707 & 0,0 & 26,8 & 71.207 & 3.500 \\
\hline & 2 & -185.850 & -185.850 & -185.850 & 0,0 & $1.571,1$ & 47.550 & 233.400 \\
\hline & 3 & -337.150 & -337.150 & -121.295 & 64,0 & $14.400,0$ & 41.050 & 378.200 \\
\hline & 4 & -337.150 & -337.150 & -120.006 & 64,4 & $14.400,0$ & 41.050 & 378.200 \\
\hline \multirow{4}{*}{ B } & 5 & -128.900 & -128.900 & -128.900 & 0,0 & $4.477,8$ & 87.700 & 216.600 \\
\hline & 6 & -70.900 & -70.900 & -70.900 & 0,0 & $2.449,2$ & 117.900 & 188.800 \\
\hline & 7 & -9.600 & -9.600 & -9.600 & 0,0 & $3.683,4$ & 157.400 & 167.000 \\
\hline & 8 & 49.100 & 49.100 & 102.328 & 109,8 & $14.400,0$ & 192.400 & 143.300 \\
\hline \multirow{4}{*}{$\mathrm{C}$} & 9 & -137.100 & -137.100 & -137.100 & 0,0 & 590,6 & 63.300 & 200.400 \\
\hline & 10 & -76.600 & -76.600 & -76.600 & 0,0 & 301,8 & 71.300 & 147.900 \\
\hline & 11 & -26.200 & -26.200 & -26.200 & 0,0 & 206,5 & 86.300 & 112.500 \\
\hline & 12 & 19.400 & 19.400 & 19.400 & 0,0 & 56,2 & 101.000 & 81.600 \\
\hline \multirow{3}{*}{$\mathrm{D}$} & 13 & -209.625 & -209.625 & -209.625 & 0,0 & $1.060,4$ & 23.775 & 233.400 \\
\hline & 14 & -162.075 & -162.075 & -135.520 & 16,4 & $14.400,0$ & 71.325 & 233.400 \\
\hline & 15 & -138.300 & -138.300 & -138.300 & 0,0 & $5.046,1$ & 95.100 & 233.400 \\
\hline
\end{tabular}

presenta que naquela coluna não houve variação de valor nas instâncias testadas. As instâncias apresentadas na Tabela 1 estão disponíveis no link https://www.dropbox. com/s/2941h4e1nhiufjq/InstanciasArtigoCharterParty.zi$\mathrm{p} ? \mathrm{dl}=0$.

Os testes computacionais foram realizados utilizando um computador Intel $i 7$ com 16 GB de memória RAM e o solver CPLEX versão 12.6, estabelecendo um tempo limite de execução de 14.400 segundos.

Na Tabela 2 são apresentados os resultados alcançados pelo CPLEX. A coluna Grupo informa o grupo da instância. A coluna Instância informa o número da instância. A coluna Função Objetivo (FO) apresentada a função objetivo que o CPLEX encontrou, sendo um valor de prêmio quando a FO for positiva, um valor de multa quando for negativa e zero quando não houver nem prêmio nem multa. Nas colunas Lower Bound (LB) e Upper Bound (UB) são apresentados os resultados do limite inferior e do limite superior quando o CPLEX não atinge a solução ótima. Utiliza-se LB para definir o resultado financeiro quando o modelo não identificar a solução ótima, pois essa seria a pior situação num modelo de maximização. A coluna GAP informa o percentual de desvio calculado pela fórmula $G A P=((L B-U B) / L B) * 100)$. A coluna Tempo de Processamento apresenta o tempo necessário para o CPLEX para chegar à solução ótima. A coluna Prêmio representa o total de prêmio e analogamente a coluna Multa representa o valor de multa de cada instância.

Um valor do GAP igual a zero representa que o CPLEX conseguiu encontrar uma solução ótima para o problema e, caso contrário, representa que o CPLEX conseguiu encontrar uma solução viável para o problema, porém sem garantia de ser a solução ótima. Com base nesta informação, pode-se verificar na Tabela 2 que o CPLEX conseguiu encontrar a solução ótima, GAP igual a zero, para 11 das 15 instâncias com um tempo de processamento médio de 1.770 segundos. As outras quatro instâncias nas quais o CPLEX encontrou uma solução viável, porém sem a garantia de ser o ótimo, GAP maior que zero, adotou-se como solução do problema o LB encontrado pelo CPLEX tendo em vista o modelo matemático ser de maximização.

Antes de iniciar as análises, vale mencionar que as mesmas consideram o resultado do porto como resultado do embarcador, pois este é o proprietário do porto e todo o planejamento do porto foi realizado para dar o melhor resultado financeiro para o embarcador/proprietário do porto.

Analisando as instâncias do Grupo A, Figura 2a, que testam o impacto do layday no cálculo de prêmio e multa, o aumento do período de layday apresenta uma tendência de redução do resultado financeiro do porto. Entende-se que o aumento do período de layday traz benefícios diretos ao armador do navio, devido à flexibilidade do período de atracação do porto. Para o embarcador, o aumento no período de layday reduz a qualidade da programação devido a imprecisão de chegada dos navios. Devido aos resultados do Grupo A e similaridade com as características do Porto de Tubarão, layday de 10 dias, definiu-se a Instância 2 como a instância referência nas análises dos outros grupos. 


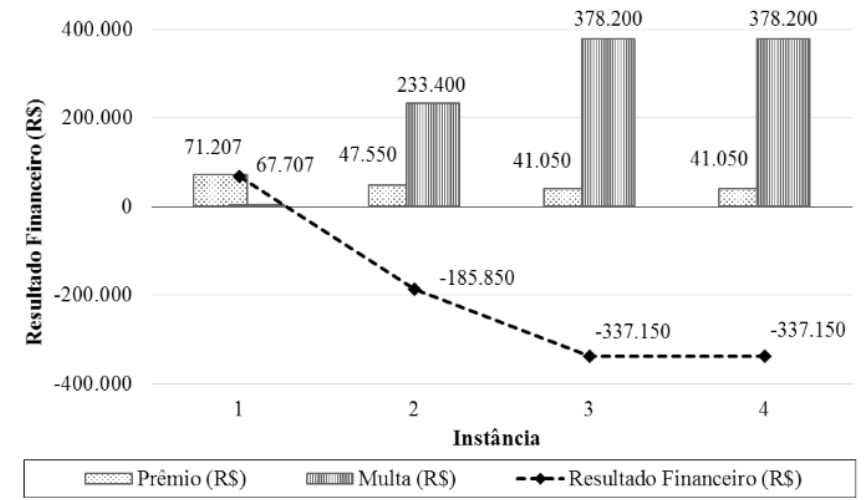

(a)

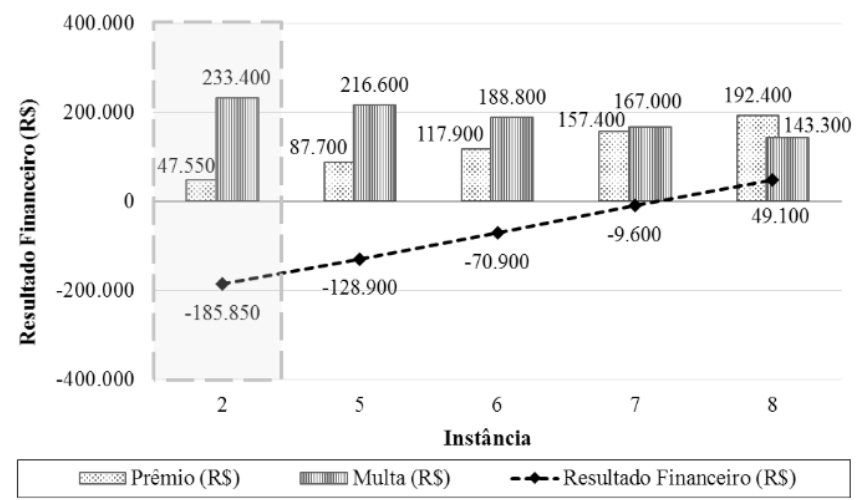

(b)

Figura 2: (a) Resultado Financeiro - Grupo A; (b) Resultado Financeiro - Grupo B

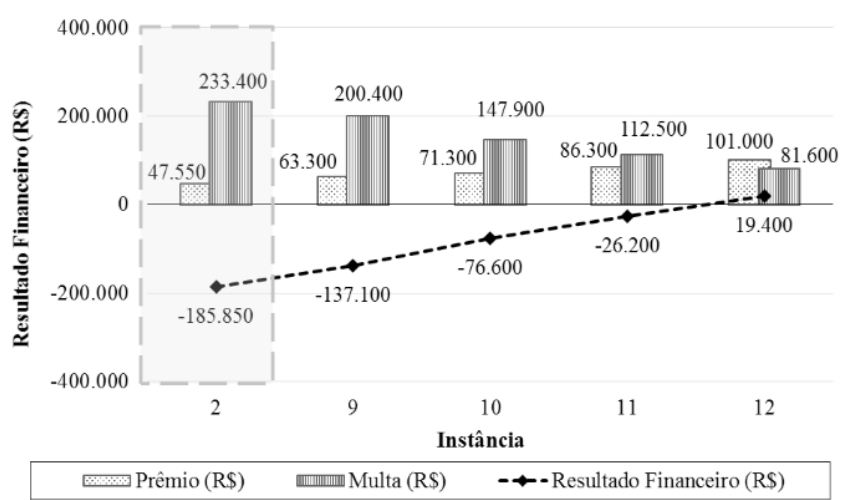

(a)

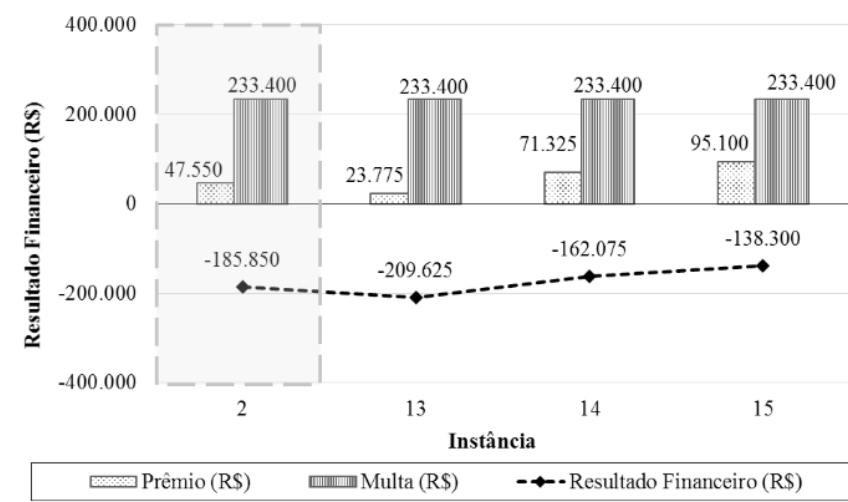

(b)

Figura 3: (a) Resultado Financeiro - Grupo C; (b) Resultado Financeiro - Grupo D

Analisando as instâncias do Grupo B, Figura 2b, que testam o impacto do laytime no cálculo de prêmio e multa, percebe-se que um aumento do laytime gera um aumento proporcional ao resultado financeiro do embarcador. Notase que o embarcador obtém melhores resultados a partir de laytime maiores.

A Instância 5, com laytime Padrão $+10 \%$, apresenta a solução ótima de R\$ 128.900 de multa e resultado financeiro negativo. Quando comparada à Instância 2, nota-se melhoria no resultado financeiro gerado pela redução da coluna Multa, mas principalmente pelo crescimento da coluna Prêmio. Por outro lado, a Instância 8, diferente as demais instâncias deste grupo, gerou a solução ótima de R \$ 49.100 de prêmio e uma melhoria no resultado financeiro maior que as demais instâncias.

Analisando as instâncias do Grupo C, Figura 3a, que testam o impacto do tempo de operação no cálculo de prêmio e multa, nota-se que foi alcançada a solução ótima em todas as instâncias, Tabela 2. O resultado financeiro é crescente nas instâncias deste grupo, onde nota-se que a melhoria é impactada pela redução no tempo de carregamento, pois quando o porto se torna mais eficiente, possivelmente ocorre à diminuição da quantidade de navios aguardando disponibilidade de berço para atracar, e ao que tudo indica, o porto gera possibilidade de operar mais navios em um mesmo horizonte de tempo, conforme visto na Figura $3 \mathrm{a}$.

Analisando as instâncias do Grupo D, Figura 3b, que testam o impacto da variação da relação percentual entre prêmio e multa, pode-se observar um aumento constante do valor de prêmio para as instâncias, Tabela 2, onde a coluna prêmio obedece a uma aparente proporcionalidade entre a variação dos valores de prêmio e a variação dos respectivos resultados financeiros em cada instância analisada. Em contrapartida o valor de multa permanece fixo.

Acredita-se que o resultado financeiro do embarcador apresente maiores variações, quando alterada a relação entre os valores de prêmio ou os valores de multa dos navios. Nota-se que a variação proporcional aplicada neste grupo para relação Prêmio e Multa, não altera a ordem de atracação dos navios, mas apenas a variação proporcional do resultado financeiro.

Em uma análise geral, analisando o impacto financeiro para o embarcador pode-se dizer que: A redução do período de Layday (Grupo A) trouxe os melhores resultados financeiros. Apesar desta redução, ela é de difícil negociação com os armadores por conta das incertezas que eles têm em relação à chegada dos navios. $\mathrm{O}$ aumento do período de laytime (Grupo B) trouxe bons resultados financeiros para o embarcador e é um fator possível de se estabelecer numa negociação junto aos armadores. A redução do tempo de carregamento dos navios (Grupo C) também trouxe bons resultados financeiros para o embarcador, no entanto, atuar no Grupo C para o porto nem sempre é simples, pois implica em novos investimentos em equipamentos e facilidades para conseguir taxas operacionais maiores. $\mathrm{O}$ aumento percentual no valor do prêmio em relação ao valor da multa 
(Grupo D) apresentou também resultado financeiro positivo para o embarcador e pode ser uma alternativa de negociação, pois é possível estabelecer uma negociação destes termos junto aos armadores.

Considerando que a abrangência do modelo matemático proposto neste artigo são os contratos de Charter Party do tipo Voyage Charter onde são definidos entre o armador e o embarcador, sendo este o proprietário do porto, períodos de layday, o laytime e ocorre a cobrança de Prêmio e Multa e também, eventualmente, os contratos Time Charter, sobretudo, dos trades de minérios, agrícolas, dentre outros, onde podem ser elaborados contratos paralelos à Time Charter para definir um período de laydays e o laytime para os navios entre o embarcador, o armador e o porto a fim de se estabelecer níveis de serviço do porto, pode-se dizer que o modelo matemático proposto atende ao embarcador, proprietário do porto, como uma ferramenta para planejamento das operações como também para análises de alternativas para apoio à tomada de decisão na negociação com os armadores, na compra de equipamentos e na ampliação de facilidades.

\section{CONCLUSÃO}

Este artigo propôs um modelo matemático para o planejamento da atracação de navios, considerando a aplicação das cláusulas de prêmio e multa do contrato Charter Party e tratando o PABD com o objetivo de aumentar o recebimento de prêmio e reduzir o pagamento de multa pelo embarcador, proprietário do porto, em função do tempo total em que os navios permanecem à disponibilidade do porto para operação.

Identificou-se por meio da revisão da literatura realizada, que até o presente momento não foi desenvolvido um modelo matemático para tratar o PABD considerando o resultado financeiro de prêmio e multa gerado nas operações portuárias. Assim, entende-se que esta é uma nova abordagem e contribui para futuros estudos sobre o PAB.

Realizou-se uma análise da variação do período de layday, aumento do período de laytime, redução do tempo de carregamento e variação no valor do prêmio sem alterar o valor da multa e pode-se concluir que para o embarcador melhorar seu resultado ele deve em uma negociação junto ao armador reduzir o período de layday, aumentar o tempo de laytime e aumentar a relação proporcional entre multa e prêmio.

Conclui-se que o modelo proposto pode atingir bons resultados no planejamento da atracação dos navios alcançando a maximização do resultado entre prêmio e multa para o embarcador e ele se mostrou como uma ferramenta eficiente para: auxílio na análise de impactos financeiros, estudos de melhoria operacional, priorização de investimentos e, inclusive, na estratégia comercial, servindo principalmente para tomada de decisão.

Como trabalhos futuros, sugerem-se: inserir na $\mathrm{FO}$ o valor da receita portuária obtida com a operação dos navios e o desenvolvimento de meta-heurísticas com o objetivo de executar instâncias de maior porte.

\section{AGRADECIMENTOS}

Os autores agradecem à FAPES (458/2013) e ao CNPq (477357/2013-0).

\section{REFERÊNCIAS}

Banos, R. S., Rosa, R. A., Mauri, G.R., Ribeiro, G.M. (2016). Modelo matemático e meta-heurística Simulated Annealing para o problema de alocação de berços com múltiplas cargas. Transportes, no prelo.

Bierwirth, C.; Meisel, F. (2010). A survey of berth allocation and quay crane scheduling problems in container terminals. European Journal of Operational Research, v. 202, n. 3, p. 615- 627. DOI: 10.1016/j.ejor.2009.05.031

Buhrkal, K., Zuglian, S., Ropke, S., Larsen, J., Lusby, R. (2011) Models for the discrete berth allocation problem: A computational comparison. Transportation Research Part E: Logistics and Transportation Review, p. 461-473. DOI: $10.1016 /$ j.tre.2010.11.016

CollyeR, W. (2006) Sobreestadia de navios: a regra once on demurrage, always on demurrage. Jus Navigandi, 1166.

Cordeau, J.F., Laporte, G., Legato, P., Moccia, L. (2005). Models and tabu search heuristics for the berth-allocation problem. Transportation Science, v. 39, n. 4, p. 526- 538.

DOI: $10.1287 /$ trsc. 1050.0120

Golias, M., Boile, M., Theofanis, S. (2006). The berth allocation problem: a formulation reflecting time window service deadlines. In: Proceedings of the 48thTransportation Research Forum Annual Meeting. Transportation Research Forum, Boston.

Golias, M. (2007). The discrete and continuous berth allocation problem: models and algorithms. Tese (Doctor of Philosophy Graduate Program in Civil and Environmental Engineering). The State University of New Jersey, New Brunswick.

Golias, M., Boile, M., Theofanis, S., Efstathiou, C. (2010). The Berth-Scheduling Problem. Transportation Research Record: Journal of the Transportation Research Board, v. 2166 , n. 1, p. 20-27.

Han, M., Li, P., Sun, J. (2006). The algorithm for berth scheduling problem by the hybrid optimization strategy GASA. In: Proceedings of the Ninth International Conference on Control, Automation, Robotics and Vision (ICARCV'06). IEEE Computer Society, Washington DC, p. 1-4.

Han, X., Lu, Z., Xi, L. (2010). A proactive approach for simultaneous berth and quay crane scheduling problem with stochastic arrival and handling time. European Journal of Operational Research, v. 207, n. 3, p. $1327-1340$.

DOI: $10.1016 /$ j.ejor.2010.07.018

Hansen, P., Oguz, C. (2003). A note on formulations of static and dynamic berth allocation problems. Les Cahiers du GERAD, 30, p. 1-17.

Imai, A., Nishimura, E., Papadimitriou, S. (2001). The dynamic berth allocation problem for a container port. Transportation Research Part B, v. 35, n. 4, p. 401-417. DOI: 10.1016/S0191-2615(99)00057-0 
Imai, A., Nishimura, E., Papadimitriou, S. (2003). Berth allocation with service priority. Transportation Research Part B. v. 37, n. 5, p. 437-457. DOI: 10.1016/S0191-2615(02)00023-1.

Imai, A., Sun, X., Nishimura, E., Papadimitriou, S. (2005). Berth allocation in a container port: using a continuous location space approach. Transportation Research Part B, v. 39, n. 3, p. 199221. DOI: $10.1016 /$ j.trb.2004.04.004

Imai, A., Nishimura, E., Papadimitriou, S. (2008). Berthing ships at a multi-user container terminal with a limited quay capacity. Transportation Research Part E, v. 44, n, 1, p. 136-151. DOI: 10.1016/j.tre.2007.03.003

Lorenzoni, L. L.; Ahonen, H.; Alvarenga, A. G. (2006). A multimode resource-constrained scheduling problem in the context of port operations. Computers \& Industrial Engineering, v. 50, n. 1, p. 55-65. DOI: 10.1016/j.cie.2005.11.001.

Mauri, G.R., Oliveira, A.C.M., Lorena, L.A.N. (2008). A hybrid column generation approach for the berth allocation problem. In: van Hemert, J., Cotta, C. (Eds.). Eighth european conference on evolutionary computation in combinatorial optimisation, v. 4972 , p. 110 - 122. DOI: 10.1007/978-3-540-78604-7_10

Mauri, G.R., Oliveira, A.C.M., Lorena, L.A.N. (2010). Resolução do problema de alocação de berços através de uma técnica de geração de colunas. Pesquisa Operacional, v. 30, n.3, p. 547562. DOI: 10.1590/S0101-74382010000300003

Meersmans, P.J.M., Dekker, R. (2001). Operations research supports container handling. Econometric Institute Report 234, Erasmus University Rotterdam.

Monaco, M.F., Sammarra, M. (2007). The berth allocation problem: a strong formulation solved by a Lagrangean approach. Transportation Science, v. 41, n. 2, p. 265-280.

DOI: $10.1287 /$ trsc. 1060.0171

Nishimura, E., Imai, A., Papadimitriou, S. (2001). Berth allocation planning in the public berth system by genetic algorithms. European Journal of Operational Research, v. 131, n. 2, p. 282-292. DOI: 10.1016/S0377-2217(00)00128-4

Praticagem ES (2015). Praticagem, o que é?. Disponível em: <www.praticagem.org.br>. Acesso em: 03 jun. 2015.

Rashidi, H., Tsang, E. (2013). Novel constraints satisfaction models for optimization problems in container terminals. Applied Mathematical Modelling, 37, p. 3601-3634.

DOI: 10.1016/j.apm.2012.07.042

Rodrigues, P. H.; Rosa, R. A.; Arpini, B. P.; Bissoli, A. L.; Ribeiro, G. M. (2015). Planejamento de atracação de navios por meio de um modelo matemático para o problema de alocação de berços contínuos com descontinuidade do cais. Transportes, v. 23, n. 1, p. 37-46. DOI: 10.4237/transportes.v23i1.681

Rosa, R. A., Resendo, L. C., Lopes, F. T. (2013). Proposta de um Modelo Matemático para o Problema de Alocação de Berços para Múltiplas Cargas (PAB-MC) com Restrições Temporais e Espaciais. In: CNT Confederação Nacional do Transporte. (Org.). Transporte em Transformação XVII. 1. ed. Brasília: Positiva, v. 1, p. 133-150.
Stahlbock, R., Voß, S. (2008). Operations research at container terminals: a literature update. OR Spectrum, v. 30, n .1, p. 1-52. DOI: 10.1007/s00291-007-0100-9

Steenken, D., Voß, S., Stahlbock, R. (2004). Container terminal operation and operations research: a classification and literature review. OR Spectrum, v. 26, n. 1, p. 3-49.

DOI: $10.1007 / \mathrm{s} 00291-003-0157-\mathrm{z}$

Stopford, M. (1997). Maritime economics. London: Routledge.

Teixeira, R. B.; Cunha, C. B. (2012). Modelo integrado para seleção de cargas e reposicionamento de contêineres vazios no transporte marítimo. Transportes, v. 20, n. 1, p. 59-70. DOI: $10.4237 /$ transportes.v20i1.442

Theofanis, S., Boile, M., Golias, M. (2007). An optimization based genetic algorithm heuristic for the berth allocation problem. IEEE Congress on Evolutionary Computation 2007 (CEC 2007). IEEE Computer Society, Washington DC, p. 44394445.

Ting, C. J.; Lin, S. W.; Wu, K. C. (2013). The Continuous Berth Allocation Problem by Simulated Annealing. In: APIEMS Conference 2013. Proceedings, Cebu, Philippines.

UNCTAD. United Nations Conference on Trade and Development (2014). Review of maritime transport 2014. New York: United Nations. p. 5. Disponível em: $<$ http://unctad.org/en/ PublicationsLibrary/rmt2014_en.pdf > . Acesso: 29 dez. 2015.

Vacca, I., Bierlaire, M., Salani, M. (2007). Optimization at container terminals: status, trends and perspectives. In: Proceedings of the swiss transport research conference (STRC). Monte Veritá/Ascona, p. 1-21.

Vale (2015). Memorial descritivo do terminal de Tubarão. Disponível em: < http://www.vale.com/pt/business/logistics/ ports-terminals/documents/pdf/memorial_descritivo terminal_tubarao.pdf $>$. Acesso em: 08 jul. 2015.

VIS, I.F.A., DE KOSTER, R. (2003) Transshipment of containers at a container terminal: an overview. European Journal of Operational Research, v. 147, n. 1, p. 1-16. DOI: 10.1016/S0377-2217(02)00293-X

Wilson, J. F. (1988). Carriage of goods by sea. London: Pitman. p. 456.

Zhou, P., Kang, H., Lin L. (2006) A dynamic berth allocation model based on stochastic consideration. In: Proceedings of the Sixth World Congress on Intelligent Control and Automation (WCICA 2006), v. 2. IEEE Computer Society, Washington DC, p. 7297-7301.

Zhou, P., Kang, H. (2008). Study on berth and quay-crane allocation under stochastic environments in container terminal. Systems Engineering - Theory \& Practice, v. 28, n. 1, p. 161169. 